\title{
Use of Syndromic Data to Determine Oral Health Visit Burden on Emergency Departments
}

\author{
Howard Burkom¹, Sherry Burrer ${ }^{1}$, Laurie Barker ${ }^{3}$, Valerie Robison ${ }^{3}$, Peter Hicks ${ }^{1}$ and Amy \\ Ising $^{2}$
}

${ }^{1}$ CDC, OSELS, Public Health Surveillance Program Office, Atlanta, GA, USA; '2University of North Carolina Department of Emergency Medicine, Chapel Hill, NC, USA; ${ }^{3}$ CDC, NCCDPHP, Div. of Oral Health, Atlanta, GA, USA

\section{Objective}

The objective was to use syndromic surveillance data from the North Carolina Disease Event Tracking and Epidemiologic Collection Tool NCDETECT and from BioSense to quantify the burden on North Carolina (NC) emergency departments of oral health-related visits more appropriate for care in a dental office (ED). Calculations were sought in terms of the Medicaid-covered visit rate relative to the Medicaid-eligible population by age group and by county.

\section{Introduction}

Concern over oral health-related ED visits stems from the increasing number of unemployed and uninsured, the cost burden of these visits, and the unavailability of indicated dental care in EDs [1]. Of particular interest to NC state public health planners are Medicaid-covered visits. Syndromic data in biosurveillance systems offer a means to quantify these visits overall and by county and age group.

\section{Methods}

Using BioSense data received from NCDETECT, 60.8 million records from 12.9 million ED visits were collected, covering all $\mathrm{NC}$ visits for state fiscal years (SFY) 2008-2010. Roughly 4\% of visits were dropped because of patient residence zip codes missing or outside NC. A careful multi-step procedure involving both dentist consultants and data analysis was used to derive classification criteria for visits whose main reason was a nontraumatic oral health problem [2]. This procedure yielded 243,970 visits by $\sim 174,600$ patients based on hospital-specific patient identifiers. Nontraumatic oral health-related visits were collected in a study set with added fields for method of payment, patient residence county, and age group. Based on previous studies, consultant preferences, and NC Medicaid eligibility guidelines, selected age groups were $0-14,15-19,20-29,30-49,50+$ years. Stratified counts of Medicaid-eligibles were obtained from the NC Dental Director by study year. Using these tables and the ED visit study set, rates of nontraumatic oral health-related Medicaid visits per 10,000 eligibles were tabulated by county and age group for each study year. Demographics of multiple-visit patients were also studied.

\section{Results}

Rates of ED oral health-related visits were substantially higher for young adults than for other age groups. From statewide rates in Table 1 , this age factor was consistent across study years.

County-level rates showed the same age pattern to varying degrees. Detailed analysis showed problem areas, with rates in 21 of 100 counties exceeding 60 per 10,000 eligibles for the 20-29 year age group. Plots and tables complemented understanding of the ED oral health visit burden by age and county. The state total ED burden for oral health problems was $\sim 2 \%(0.2 \%-9.7 \%$ by county $)$.

\section{Conclusions}

Judicious use of syndromic data with external information, such as the detailed Medicaid denominators and the Method of Payment codes for each visit above, can give quantified estimates for policyrelated public health issues. In the current study, the derived oral health visit rates gave numerical detail to concerns about the use of NC EDs for nontraumatic oral health problems by low-income persons affected by the economic recession. Results also show rate variation by county and can be combined with access-to-care data to inform planning of effective local measures to improve access to dental services and thus reduce the ED visit burden.

Table 1. NC Statewide Oral Health Medicaid Visits to Emergency Departments per 10,000 Eligibles

\begin{tabular}{|c|c|c|c|}
\hline Visits per 10,000 Eligibles & SFY 2008 & SFY 2009 & SFY 2010 \\
\hline All Age & 9.5 & 9.9 & 9.2 \\
\hline $0-14$ yrs & 1.9 & 1.8 & 1.8 \\
\hline $15-19 \mathrm{yrs}$ & 8.4 & 9.0 & 7.9 \\
\hline $20-29 \mathrm{yrs}$ & 42.6 & 43.4 & 39.6 \\
\hline $30-49 \mathrm{yrs}$ & 22.9 & 24.2 & 22.5 \\
\hline $50+\mathrm{yrs}$ & 9.5 & 2.5 & 2.4 \\
\hline
\end{tabular}

\section{Keywords}

emergency department; chief complaint; BioSense; oral health; NCDETECT

\section{Acknowledgments}

Mark Casey, North Carolina Dental Director for Medicaid eligibility and care provider data; Chris Okunseri, Marquette University for case definition advice; Lana Deyneka of the North Carolina Department of Health and Human Services for consultation; Dennis Falls of NCDETECT for Method of Payment information

\section{References}

1. Shortridge, E. and Moore, J. 2010. "Use of Emergency Departments for Conditions related to Poor Oral Health Care." Final Report. Washington, DC: Office of Rural Health Policy. August 2010.

2. Burrer S, Burkom H, Okunseri C, Barker L, Robison V, Nontraumatic Oral Health Classification developed from Syndromic Data, Abstract, International Society of Disease Surveillance Conference 2012.

\footnotetext{
*Howard Burkom

E-mail: howard.burkom@jhuapl.edu
} 